\title{
Assessing the Quality of the After-Visit Summary (AVS) in a Primary-Care Clinic
}

\author{
Tasaduq Hussain Mir, MD, Amimi Osayande, MD, Kimberly Kone, MD, \\ Kate Bridges, $B A$, and Philip Day, $P h D$
}

Background and Objective: As part of Affordable Care Act, the Centers for Medicaid Services (CMS) recommend physicians provide patients with an After-Visit Summary (AVS) following a clinic visit. Information should be relevant and actionable with specific instructions regarding their visit and health. Until recently, this recommendation was included as part of meeting the standard for Stage 1 Meaningful Use for all physicians using electronic-health-record (EHR) technology. In 2016, CMS issued a Notice of Proposed Rulemaking to institute parts of the Medicare Access and CHIP Reauthorization Act of 2015 Merit-based Incentive Payment System, which continues to focus on quality, resource use, and use of certified EHR technology. The purpose of this study was to assess the usefulness of the AVS for patients seen at the Parkland Family Medicine Residency Clinic.

Methods: Electronic medical records of 250 randomly selected patients seen at the Parkland Family Medicine Residency Clinic between July 2013 and July 2014 were reviewed using the 3 W's question format, a modified version of the National Patient Safety Foundation's "Ask Me 3 Program," designed to improve communication between patients and their health care providers.

Results: The goal of the quality improvement study was to ensure that all patients receive a meaningful (relevant, accurate, and actionable) AVS after each clinic visit. Chart review indicated that $100 \%$ of patients received an AVS after each clinic visit. Of these patients, $51.2 \%$ were Spanish speaking, $47.2 \%$ English speaking, and 1.6\% spoke neither English nor Spanish. Of the non-English-speaking patients, 84.8\% received the AVS in their first language; the other $15.2 \%$ received the AVS in English. Sixteen percent (16\%) of patients overall were considered to have received a nonmeaningful AVS. Reasons for the AVS not being meaningful included not containing any information on the patient's presenting problem (39.2\%), physician intervention (35\%), or plan of care $(18.4 \%)$.

Conclusions: This study confirmed that although we demonstrate meaningful use of our EHR system, the content of the AVS needs to be improved on. (J Am Board Fam Med 2019;32:65-68.)

Keywords: Electronic Health Records, Meaningful Use, Primary Health Care, Quality Improvement

The Centers for Medicaid Services (CMS) recommend physicians provide patients with an AfterVisit Summary (AVS) following a clinic visit. ${ }^{1}$ In this AVS, information should be relevant to pa-

\footnotetext{
This article was externally peer reviewed.

Submitted 14 February 2018; revised 29 August 2018; accepted 1 September 2018.

From the Department of Family and Community Medicine, University of Texas Southwestern Medical School, Dallas, TX (THM); Family Medicine Residency Program, Gwinnett Medical Center, Lawrenceville, GA (AO); Department of Family and Community Medicine, University of Texas Health Sciences Center-San Antonio, San Antonio, TX (KK); Department of Family and Community Medicine, University of Texas-Southwestern Medical School, Dallas $(\mathrm{KB}, \mathrm{PD})$.

Funding: none.
}

tients and actionable with specific instructions regarding their visit and health. Until recently, this recommendation was necessary to meet the standards for Stage 1 Meaningful Use for all physicians using electronic health records (EHRs). On April

Conflict of interest: none declared.

Prior Presentation: Assessing the Quality of the After Visit Summary (AVS) in a Residency-Based Primary Care Clinic. Presented at the John Peter Smith 9th Annual Research Day Empowerment through Research and Education, June 5, 2015, Fort Worth, TX.

Corresponding author: Tasaduq Hussain Mir, MD, Department of Family and Community Medicine, University of Texas-Southwestern Medical School, 5323 Harry Hines Boulevard, Dallas, TX 75390-9165 (E-mail: Tasaduq.Mir@utsouthwestern.edu). 
27, 2016, CMS issued a Notice of Proposed Rulemaking to institute parts of the Medicare Access and CHIP Reauthorization Act of 2015 (MACRA) Merit-based Incentive Payment System, which continues to focus on quality, resource use, and use of certified EHR technology, which includes the AVS. The proposed rule is based on input from patients, caregivers, physicians, clinicians, and health-care professionals. ${ }^{2}$

Studies have confirmed that patients and physicians find the information in the AVS useful. ${ }^{3}$ However, to our knowledge, there have been no studies to assess the quality of the AVS in a Family Medicine Residency program. The purpose of this exploratory study is to assess the usefulness of the AVS for patients and the areas that need improvement for best practice. In a CMS affiliated program, residents are expected to document their patient health information and provide their patients with an AVS with the aid of the EHR system. At the time of the study, the number of patients who actually received an AVS or the quality of what they received was unknown. Along with knowing how many patients are receiving an AVS, it is essential to understand if the AVS provides meaningful information and if it addresses the salient issues for which the patient sought medical help.

\section{Methods}

This study was reviewed by the Institutional Review Board and deemed exempt. This retrospective cohort study consisted of 250 charts from distinct adult patients seen at least once over a 12 -month period from July 2013 to July 2014 in the Family Medicine Residency Clinic at Parkland Heath and Hospital System (PHHS). The study team requested data from PHHS and the data engineers returned 250 charts from this time period. Selected charts included patients over the age of 18 years and both sexes. There were no exclusions based on health condition or presentation. Data points from the chart were patient preferred language listed as primary language in EHR, language AVS was written, presenting problem, physician intervention, and plan of care.

Electronic health records (EHR) of these patients were reviewed and coded by faculty and a resident using the 3 W's question format, a modified version of the National Patient Safety Foundation's “Ask Me 3 Program," designed to improve communication between patients and their healthcare providers. ${ }^{4}$ This program was chosen because it has proved successful in promoting patient-provider interaction in previous studies. ${ }^{5-8}$ The information was dichotomized based on whether the information was present or absent from the EHR, and the meaningfulness of the AVS was assessed by its ability to provide patients with the answers to the following "Ask Me 3 Program" modified questions:

1. What did the patient present with? (presenting problem)

2. What did the physician do for the patient? (physician intervention)

3. What does the patient need to do before the next visit? (plan of care)

In the original CMS AVS requirements there are 11 components that should be addressed for meaningful use. ${ }^{1}$ Most of these components are autopopulated by the EHR, such as clinic contact information and patient name, but the patient's presenting problem, doctor's intervention, and patient's instructions would not be automatically part of the AVS unless documented by the provider. All 3 questions above from the 3 W's align with elements of the CMS requirements and were considered the most useful AVS components for the patient, therefore all 3 were necessary to be considered meaningful. Descriptive statistics and $\chi^{2}$ analyses were conducted using SPSS Statistics 25 (IBM Corp., Armonk, NY) software to determine prevalence and significance.

\section{Results}

The goal of this study was to ensure that all patients receive a meaningful-relevant, accurate, and actionable-AVS after each clinic visit. Charts reviewed $(n=250)$ indicated that $100 \%$ of patients received an AVS after each clinic visit. A majority of these patients spoke Spanish as their primary language $(51.2 \%)$ and most patients received an AVS in their primary language (82\%) (Table 1). Patients who spoke primarily English were more likely to receive their AVS in their primary language $(97.5 \%)$ than Spanish speakers (70.3\%). However, there was greater error in delivery of the AVS to the English-speaking patients. When the AVS was written in English, $73.5 \%$ of English 
Table 1. After-Visit Summary (AVS) Descriptive Information

\begin{tabular}{lc}
\hline & No. $(\%)$ \\
\hline Patient primary language & \\
Spanish & $128(51.2)$ \\
English & $118(47.2)$ \\
Other & $4(1.6)$ \\
AVS language & \\
Spanish & $93(37.2)$ \\
English & $157(62.8)$ \\
Primary problems & \\
AVS included & $152(60.8)$ \\
AVS excluded & $98(39.2)$ \\
Visit plans & \\
AVS included & $161(64.4)$ \\
AVS excluded & $89(35.6)$ \\
Plan of care & \\
AVS included & $204(81.6)$ \\
AVS excluded & $46(18.4)$ \\
\hline
\end{tabular}

AVS, after-visit summary.

AVSs went to an English speaker. On the other hand, an AVS written in Spanish had a greater chance of going to a recipient who was primarily Spanish speaking (96.8\%).

Interestingly, an AVS in Spanish was significantly more likely to contain information about a primary problem whereas those with English AVS were less likely $\left(\chi^{2} 250=36.23 ; P=.000\right)$. This same relationship between language and information presented was consistent when considering the information for physician intervention $\left(\chi^{2} 250=\right.$ $24.49, P=.000)$ and plan of care $\left(\chi^{2} 250=16.73\right.$; $P=.000)$. See Table 2 .

The 3 questions from the Ask Me 3 Program were aligned with 3 potential characteristics of an AVS as fulfillment responses to those 3 questions to derive measurable aspects of an AVS's meaningful- ness. If an AVS addressed the patient's main reason for the visit then it is considered relevant; if an AVS correctly addresses the physician's intervention then it is considered accurate; and finally, if an AVS describes the patient's required actions (fill a prescription, see a specialist, etc.) then an AVS is considered actionable. Sixteen percent $(16 \%)$ of patients overall were considered to have received a nonmeaningful AVS. Reasons for the AVS not being meaningful included not containing any information on the patients' presenting problem (39.2\%), physician intervention (35.6\%), or plan of care (18.4\%).

\section{Discussion}

Although there is no agreement on what is relevant and actionable data when it comes to the AVS, studies show primary-care patients prefer to receive an AVS and the amount of information included does not affect content recall or satisfaction with the information. ${ }^{9}$ Studies also show that the AVS does not serve as a communication tool to engage patients. ${ }^{10}$ Our study confirmed that although all patients in our clinic received an AVS after every clinic encounter, it was not always meaningful due to lack of information put into the freeform portion of the EHR by the resident. For us, AVS not being meaningful means that either 1 or all "Ask Me 3 Program" modified questions were not addressed. For instance, a high number of Spanish-speaking patients received an AVS in English, which does not meet Meaningful Use regulations' goal for the AVS. If the visit was conducted in English with a primarily Spanish speaking patient, the provider may have a greater likelihood of producing an English AVS.

In addition, if patients received their summary in Spanish, they were significantly more likely to re-

Table 2. Percent of Patients Who Received the Following Information in Their After-Visit Summary (AVS) and on Language of AVS

\begin{tabular}{|c|c|c|c|c|c|c|}
\hline \multirow{3}{*}{ Language of AVS } & & & \multicolumn{4}{|c|}{ Information Presented in AVS } \\
\hline & \multicolumn{2}{|c|}{ Primary Problems (\%) } & \multicolumn{2}{|c|}{ Visit Plans (\%) } & \multicolumn{2}{|c|}{ Plan of Care (\%) } \\
\hline & Yes & No & Yes & No & Yes & No \\
\hline & \multicolumn{2}{|c|}{$\chi^{2}(250)=36.23^{*}$} & \multicolumn{2}{|c|}{$\chi^{2}(250)=24.49^{*}$} & \multicolumn{2}{|c|}{$\chi^{2}(250)=16.73^{*}$} \\
\hline English & 46.5 & 53.5 & 52.9 & 47.1 & 73.9 & 26.1 \\
\hline Spanish & 84.9 & 15.1 & 83.9 & 16.1 & 94.6 & 5.4 \\
\hline
\end{tabular}

${ }^{*} P<.001$. 
ceive more complete information, including information concerning presenting problem, physician intervention and treatment. Spanish-speaking patients prefer to see Spanish-proficient resident physicians, who may be more careful or able to write more details in the AVS.

Though we emphasize the importance of our findings in the usefulness of the information given to patients, we acknowledge limitations to this study such as patient's ability to speak more than one language (bilingual) or their proficiency in English as a second language because the EHR only lists one primary language. Documenting these characteristics could potentially affect the outcomes of the study as both factors are likely to decrease the discrepancy noted between the patient's language and the language on the AVS provided. In some cases, an English AVS may be appropriate for a primarily Spanish speaking patient if they have high English proficiency. The presentation of AVS information should be further researched along with educational interventions for keeping the content of AVSs consistent and meaningful.

\section{Conclusion}

Potential barriers that impede a meaningful AVS in the present clinic are likely to be similar to those encountered by primary-care providers across the nation, such as the amount of time required keeping the problem and medication lists current or the language used in the AVS. Another barrier to patients receiving an AVS is the language barrier between staff or residents and patients. The barriers to providing an accurate AVS may be evaluated in future studies. Further studies are needed to determine whether a meaningful AVS improves patients' chronic-care outcomes and the study team is currently undertaking such a project that will also address patient perception and understanding of AVS and health literacy. Although CMS no longer mandates that AVS are provided to all patients, we believe it has the potential to increase patientphysician communication. Patient compliance and adherence may be improved by providing a meaningful AVS, so it is essential to provide patients this opportunity through clear follow-up information.
To see this article online, please go to: bttp://jabfm.org/content/ 32/1/65.full.

\section{References}

1. Office of the National Coordinator for Health Information Technology. Providing clinical summaries to patients after each office visit. Available from: www.healthit.gov/sites/default/files/measure-tools/ avs-tech-guide.pdf. Accessed August 10, 2018.

2. Department of Health and Human Services. Medicare program; Merit-based Incentive Payment System (MIPS) and Alternative Payment Model (APM) incentive under the physician fee schedule, and criteria for physician focused payment models. Available from: https://www.gpo.gov/fdsys/pkg/FR2016-05-09/pdf/2016-10032.pdf. Accessed March 29, 2018.

3. Federman AD, Sanchez-Munoz A, Jandorf L, Salmon C, Wolf MS, Kannry J. Patient and clinician perspectives on the outpatient after-visit summary: A qualitative study to inform improvements in visit summary design. J Am Med Info Assoc 2017;24(e1): e61-e68.

4. National Patient Safety Foundation. Ask me 3: Good questions for your good health. Institute of Healthcare Improvement Web site. Available from: http:// www.npsf.org/?page $=$ askme3. Accessed March 29, 2018.

5. Mika VS, Wood PR, Weiss BD, Treviño L. Ask Me 3: Improving communication in a Hispanic pediatric outpatient practice. Am J Health Behav 2007; 31(Suppl 1):S115-S121.

6. Baijens SWE, Huppelschoten AG, Van Dillen J, Aarts JWM. Improving shared decision-making in a clinical obstetric ward by using the three questions intervention, a pilot study. BMC Pregnancy Childbirth 2018;18:283.

7. Miller MJ, Abrams MA, McClintock B, et al. Promoting health communication between the community-dwelling well-elderly and pharmacists: The Ask Me 3 program. J Am Pharm Assoc (2003) 2008;48: 784-92.

8. Grice GR, Tiemeier A, Hurd P, et al. Student use of health literacy tools to improve patient understanding and medication adherence. Consultant Pharm 2014;29:240-53.

9. Pavlik V, Brown AE, Nash S, Gossey JT. Association of patient recall, satisfaction, and adherence to content of an electronic health record (EHR)-generated after visit summary: A randomized clinical trial. J Am Board Fam Med 2014;27:209-18.

10. Neuberger M, Dontje K, Holzman G, Corser B, Keskimaki A, Chant E. Examination of office visit patient preferences for the after-visit summary (AVS). Perspect Health Inf Manag 2014;11:1d. 